\title{
Accurate Signal Timing from High Frequency Streaming Data
}

\section{Jonathan Wang ${ }^{1}$, Alex Sim${ }^{2}$, K. John $\mathrm{Wu}^{2}$, Seongwook Hwangbo ${ }^{3}$}

${ }^{1}$ Rice University, ${ }^{2}$ Lawrence Berkeley National Laboratory, ${ }^{3}$ Hyundai Electric \& Energy Systems Co., Ltd.

\section{RESEARCH GOAL}

To analyze massive high-frequency streaming data to localize the coordinates of partial discharge events by identifying the signal arrival time in noisy data

\section{BACKGROUND}

- Insulation failure is the most common type of transformer breakdown

- Partial Discharge (PD), an internal arcing event, is a symptom of insulation failure

- Induced PD at 2 locations: PD1 and PD2

- Streaming PD signal data

- Collected by ultra-high frequency (UHF) sensors

- $300 \mathrm{MHz}-1 \mathrm{GHz}$ frequency

- 0.4 ns per data point, 12 GB per sec

- 4 channels per sample (4 sensors)

- Challenge: Use PD voltage data to locate source of PD

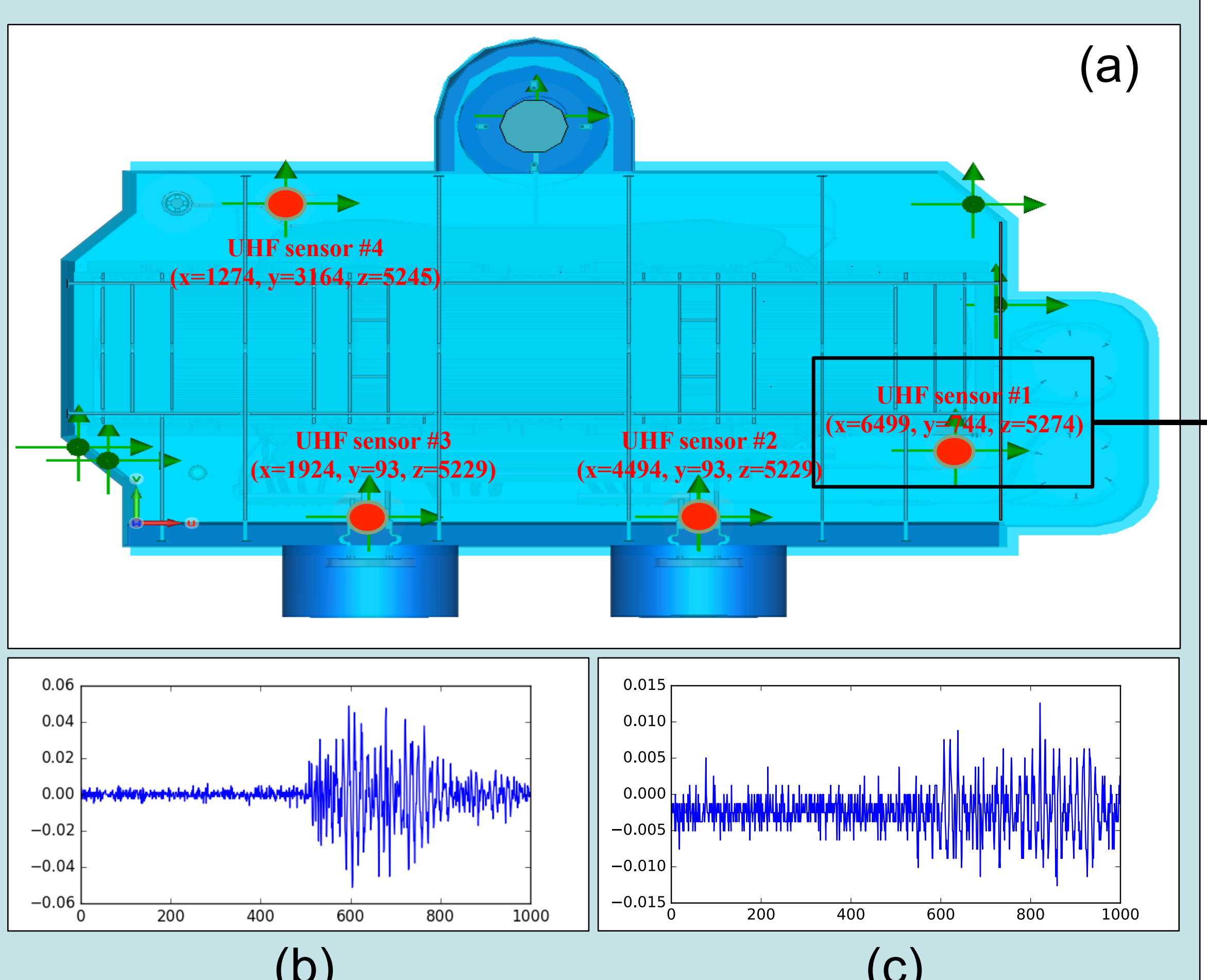

(b)

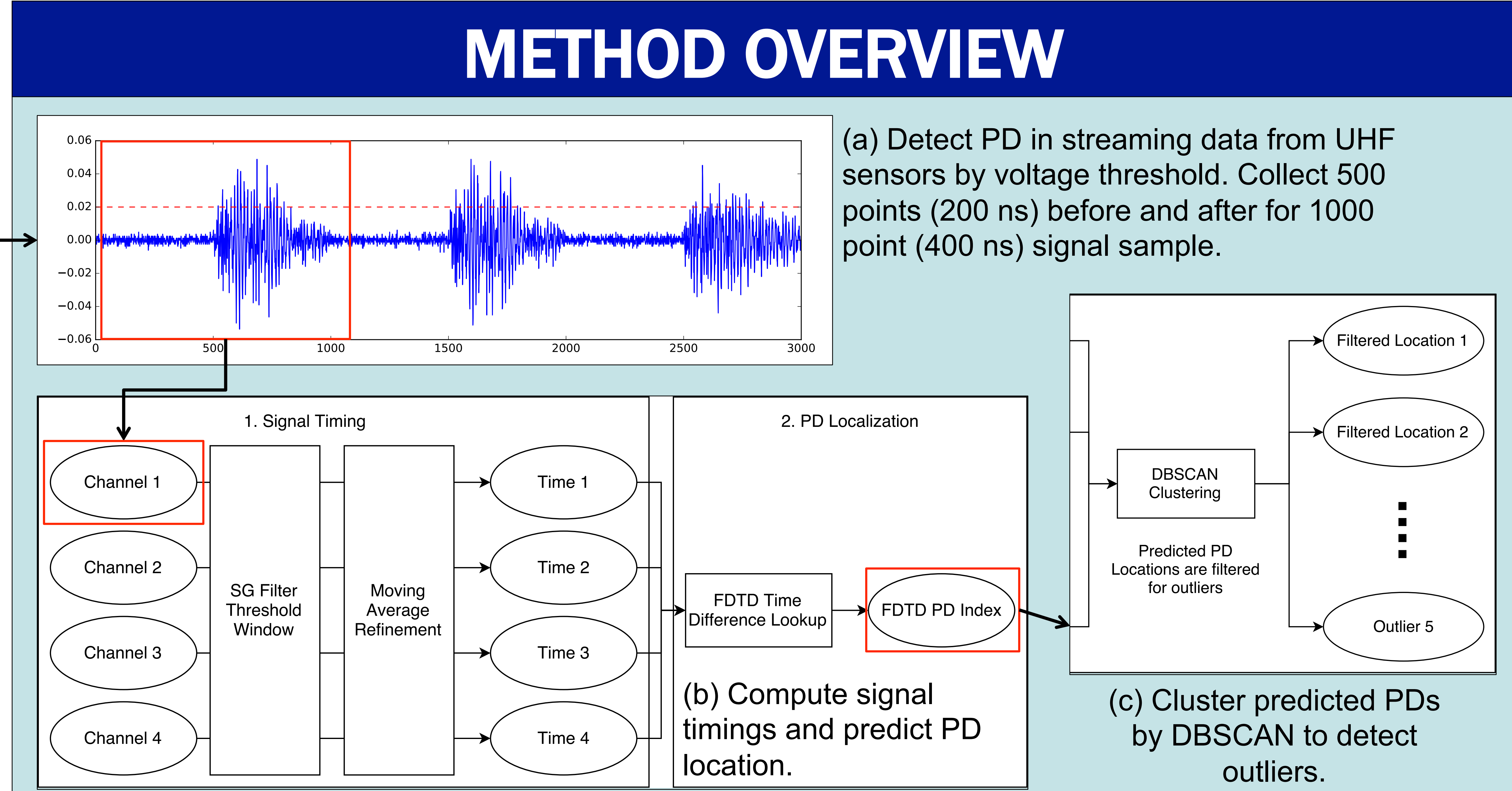

Figure 2: (a) Obtain PD signal sample from streaming data. (b) For Signal Timing: de-noise data with Savitzky-Golay Filter and find arrival window using threshold. Refine window with moving average and threshold to obtain arrival time. For PD Localization: use 4 signal arrival times to lookup closest FDTD index. (c) Remove outliers from set of all samples.

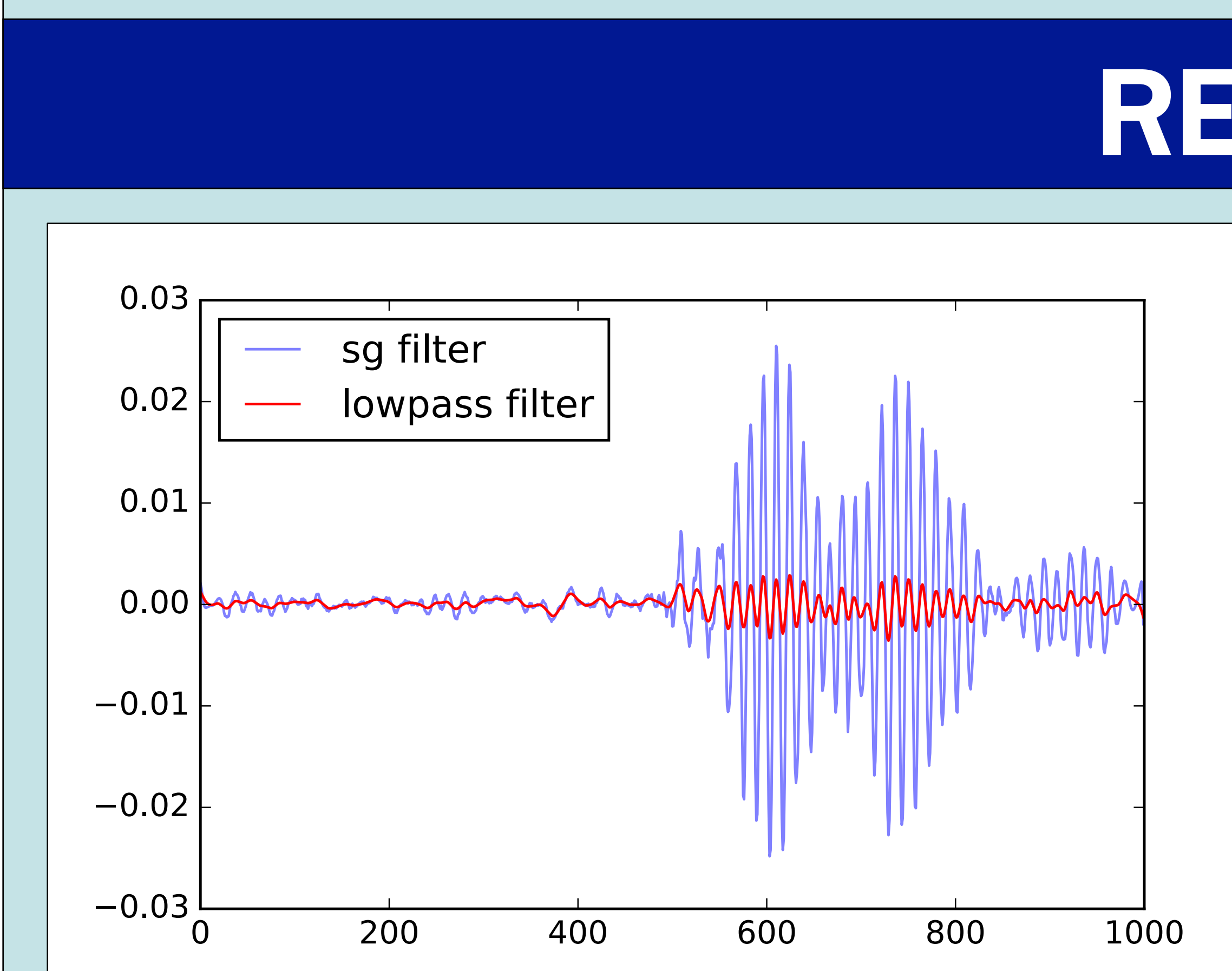

Figure 3: In low noise data, the Savitzky-Golay Filter (SG Filter) results in a significantly higher signal-to-noise ratio (SNR) than the lowpass filter, making signal detection trivial.

Relatively easy to distingurs sensor diagram. (b) Relatively easy to distinguish signal arrival in low noise data (c) but much harder in high noise data where signal arrival is obscured by noise.

\section{SIGNAL TIMING}

- Distinguish signal from noise

- Difficult with low signal-to-noise ratio - Savitzky-Golay Filter with Refinement

- Filter noisy data by convolution to improve signal-to-noise ratio (SNR)

- Preserves features better than other filters - Determine window of signal arrival

- Refine window with moving average - Define threshold based on noise

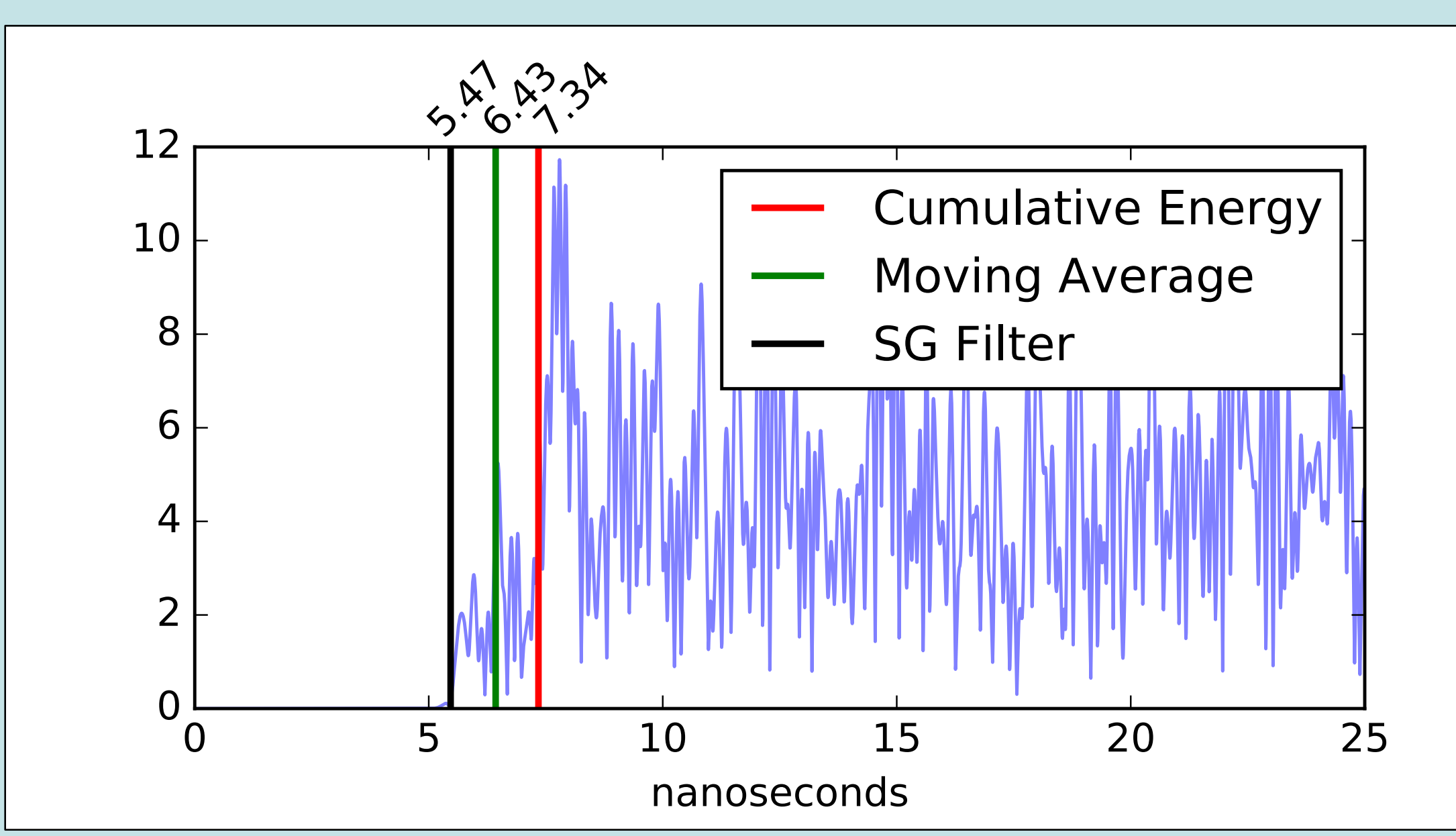

Figure 5: SG Filter signal timing method identifie signal arrival time $2 \mathrm{~ns}(0.5 \mathrm{~m})$ closer than

cumulative energy, the current timing method. The signal arrival is where the signal becomes non-
PD LOCALIZATION

- Use signal arrival times to find PD location - Multilateration

- Solve 4 quadratic equations for $\mathrm{x}, \mathrm{y}, \mathrm{z}, \mathrm{t}$

- Assumes signals travel in straight line

\begin{tabular}{|l|l} 
sensor & Figure 7: Times for
\end{tabular}

sensor
signal to reach sensor
(in upper left corner). PD
ans
s.

W straight lines due to transformer internal
structure.

\section{FDTD Lookup}

- Divide transformer into $30 \mathrm{~cm}$ cubes

- Simulate propagation of PD signal from

each index to each sensor

- Record timings in lookup table

- Find FDTD index with minimum time error

$$
\arg \min _{k} \sqrt{\sum_{i \in\{1,3,4\}}\left(T D_{i, 2}-P D_{i, 2}^{(k)}\right)^{2}}
$$

Validation: Simulate PD at each FDTD index Find distance between simulated point and predicted point

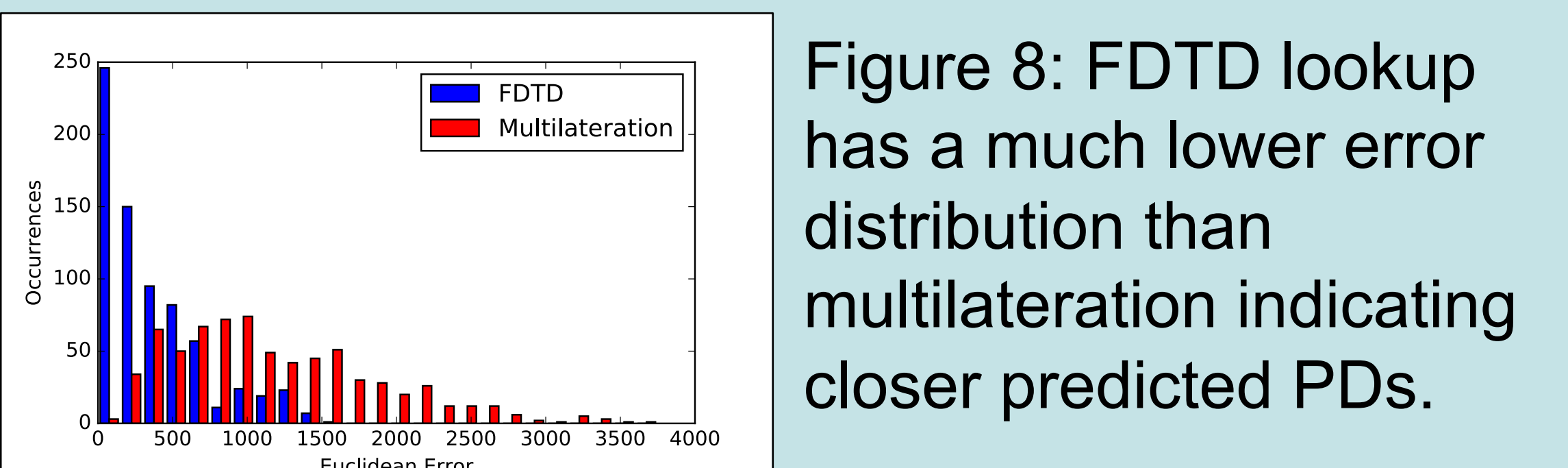

\section{CONCLUSION}

- Savitzky-Golay filter method with refinement + FDTD lookup

- $2 \mathrm{~ns}$ timing improvement $\approx 0.5 \mathrm{~m}$

- Localization improvement with SG

Filter (using FDTD)

- From $91 \%$ to $95 \%$ in PD1 case

(high signal-to-noise ratio)

- From $13 \%$ to $48 \%$ in PD2 case

(low signal-to-noise ratio)

\section{ACKNOWLEDGMENTS}

This work was supported by the U.S. Department of Energy, under Contract No. DEAC02-05CH11231.

Figure 6: Using SG Filter signal timing method, (a) localization results with multilateration are scattered and far from actual PDs with only 33\% accuracy for the above case. (b) Localization with FDTD has $95 \%$ accuracy and is much more consistent.

\section{CONTACT}

Jonathan Wang

Rice University

Rice University SRICE 